\title{
Scaling laws in bacterial genomes: A side-effect of selection of mutational robustness?
}

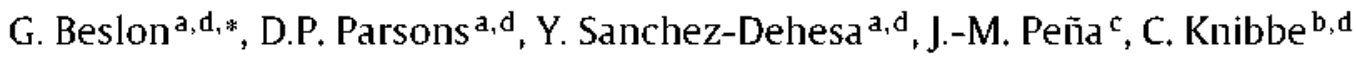 \\ a Université de Lyon, CNRS, INSA-Lyon, LIRIS, UMR5205, f-69621, france \\ b universite de Lyon, CNRS, Universite Lyon 1, LIRS, UMR5205, F-69622, France \\ - DATSi, universidad Poiltécnica de Madrid, Spain

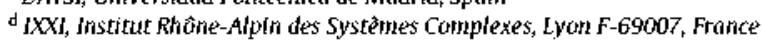

A R T I C L E I N F O

Keywords:

Modelling

Scaling laws

Gene content

Transcription Factors

Mutational robustness

Evolvability

\begin{abstract}
A B S T R AC T
In the past few years, numerous research projects have focused on identifying and understanding scaling properties in the gene content of prokaryote genomes and the intricacy of their regulation networks. Yet and despite the increasing amount of data available, the origins of these scalings remain an open question. The RAevol model, a digital genetics model, provides us with an insight into the mechanisms involved in an evolutionary process. The results we present here show that (i) our model reproduces qualitatively these scaling laws and that (ii) these laws are not due to differences in lifestyles but to differences in the spontaneous rates of mutations and rearrangements. We argue that this is due to an indirect selective pressure for robustness that constrains the genome size.
\end{abstract}

\section{Introduction}

Despite the huge diversity of living beings - from the smallest life forms to the biggest trees or mammals - some allometric ratios have been shown to be remarkably conserved among the living kingdom. For instance, it has been shown that various physiological characteristics of all organisms scale with their body mass and follow simple power-law behaviors whose exponents are multiples of $1 / 4$ (West et al., 2002). These scaling laws may reveal some fundamental principles of life, typically the necessity, for all organisms, to distribute energy and nutrients efficiently within their whole body (West and Brown, 2005).

At the molecular level, the ever-increasing number of sequenced genomes allows largescale comparative analysis. This analysis has revealed that several molecular traits also follow characteristic scaling laws. For instance, the genome size has been shown to scale as a power-law of the spontaneous mutation rate in DNAbased microbes (Drake, 1991; Drake et al., 1998). More recently, different genomic properties have been shown to follow power-law distributions (Luscombe et al., 2002; Koonin et al., 2002).

In prokaryotes, genomic structures can be very diverse, with genome sizes ranging from $\sim 500 \mathrm{~kb}$ for the endosymbiont Buchnera aphidicola (Viñuelas et al., 2007) to more than $6 \mathrm{Mb}$ for Pseudomonas aeruginosa (Stover et al., 2000). Similarly, the number of genes ranges from a few hundred ( $\sim 600$ for $B$. aphidicola) to more than 5500 for $P$. aeruginosa. Variations in the functional content of the genomes are also visible at the transcription level: some organisms (e.g., B. aphidicola) are hardly able to regulate their transcriptional activity (Reymond et al., 2006) while others display complex regulation networks made up of thousands of tightly interconnected nodes (Stover et al., 2000). When the sequenced bacterial genomes are considered globally, the diversity of genomic structure in prokaryotes is even more striking. Through the analysis of the annotated sequences, it was shown that the number of genes in each functional category scales as a power-law of the total number of genes in the genome and that the exponent of this law depends on the functional role of the family: the number of transcription factors (TFs), in particular, scales quadratically with the total number of genes while metabolic genes scale at most linearly with it (van Nimwegen, 2003: Molina and van Nimwegen, 2008). Moreover, this increase is also correlated with the size of the genome (Konstantinidis and Tiedje, 2004). These results suggest that the intricacy of regulation networks grows faster than the size of the network itself.

The question of the origin and universality of such scaling laws remains open (Cordero and Hogeweg, 2007; Molina and van Nimwegen, 2009). Some evolutionary models based on gene duplication and deletion can produce power-law relations (Luscombe et al., 2002; Foster et al., 2006) but these models directly consider 


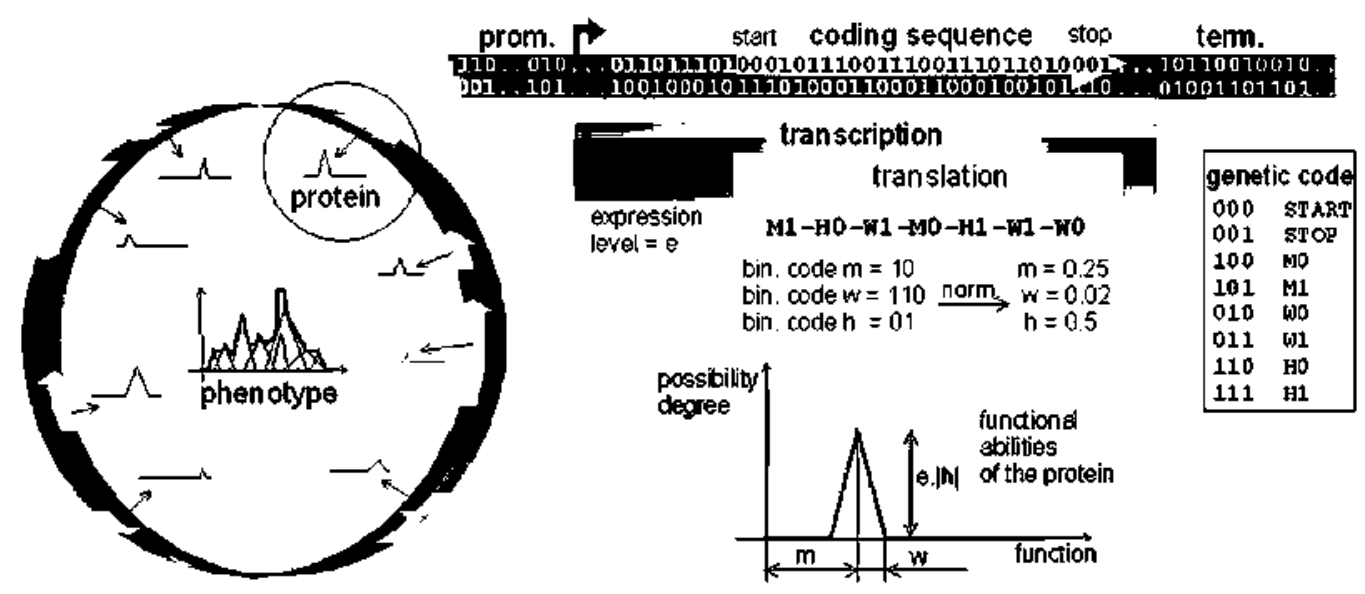

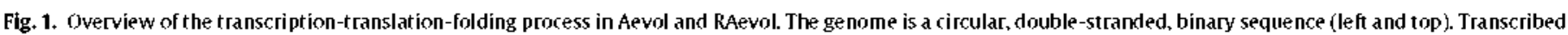

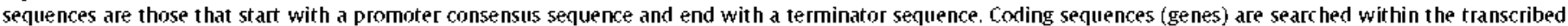

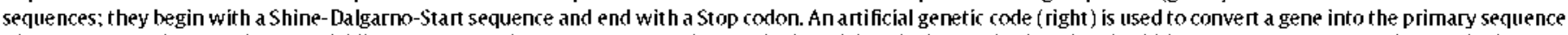

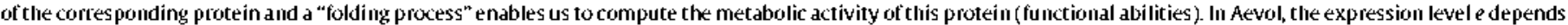

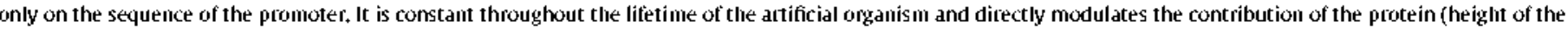

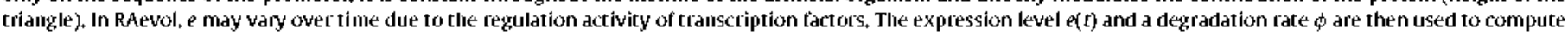

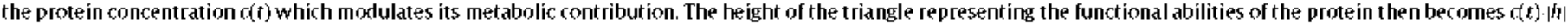
(see Section 5 and Eq. (2)).

the mutations that went to fixation in the population, without distinguishing the respective influences of the various underlying processes - genetic drift, natural selection, mutational biases. However, the classical hypothesis is that the scaling has a selective origin. It is often assumed that these scaling laws result from a selection process linked to bacterial lifestyle: complex environments would require the coordination of multiple metabolic pathways (Cases et al., 2003). Alternatively, it has been argued that any increase in the genetic repertoire of an organism (e.g., a new metabolic pathway) generates a need for new transcription factors in order to regulate its activity within the existing metabolism (Maslov et al., 2009).

Actually, despite the tremendous advance in the fields of genomics and transcriptomics, it is still not clear whether these "molecular allometric laws" result from selective constraints (e.g.* selection for short genomes or integrated networks), from the intrinsic dynamics of the evolutionary process or from any other mechanism still to be revealed (Molina and van Nimwegen, 2009).

In order to explore the evolutionary pressures on the genomic and transcriptomic structures and their dependence on external conditions (e.g., environmental conditions, population size, selection strength, mutation rates), an interesting approach is to use digital genetics models (Adami, 2006) where a finite population of virtual organisms is explicitly simulated in a virtual environment. These "organisms" are complex enough to be analyzed in terms of molecular structure but they are also simple enough to allow for the computation of a fitness value, based on their genetic sequences and on the virtual environment. It is hence possible to implement a selection procedure. In such models, the evolutionary forces are precisely tuned and it is possible to test experimentally how they shape the structure of the organisms.

Digital genetics has already shown that Darwinian evolution can have counter-intuitive effects, due to indirect selective pressures on variability. lndeed, since the mutational variability of the phenotype is partly under genetic control there can be a polymorphism in the level of variability in a population. Moreover, the variability level can influence the survival of lineages: those with inappropriate levels of variability can go to extinction due to a lack of robustness or evolvability - defined as the capacity of a lineage to generate adaptive heritable genotypic and phenotypic variation (Nehaniv, 2005). Thus there can be an indirect selective pressure on the factors that control the mutational variability of the phenotype: the mutation rate (Sniegowski et al., 2000), but also the properties of the genotype-phenotype map like modularity (Wagner and Altenberg, 1996).

Such indirect pressures are difficult to unravel in real organisms. Yet they can easily be studied using digital genetics experiments. For example, it was shown that, under high mutation rates, the indirect selection for mutational robustness can be strong enough to overcome the direct selection of immediate fitness, an effect called "survival of the flattest" (Wilke et al, 2001). It was also shown that a specific gene order can evolve by indirect selection of robustness against crossing-over (Pepper, 2003).

In this paper, we propose an integrated model of the evolution of regulatory networks, where the network level is not considered on its own but as a key layer between the genome sequence (where the mutations occur) and the phenotype (on which selection acts). We present our first large campaign of in silico experimental evolution with this model. Our results show that the model reproduces some known allometric laws, enabling us to propose hypotheses regarding their origin.

\section{RAevol in a nutshell}

To study the evolution of the structure of genomes and gene networks, we have developed an integrated model, RAevol (Regulatory-Aevol). This model extends the Aevol model (Artificial evolution), previously developed in our team to study robustness and evolvability in artificial organisms (Knibbe et al., 2007a,b, 2008). We provide here an overview of the RAevol model. A detailed description of the model is available in Section 5.

In both Aevol and RAevol, each artificial organism owns a genome whose structure is inspired by prokaryotic genomes. It is organized as a circular double-strand binary string containing a variable number of genes separated by non-coding sequences (Fig. 1). A set of pre-defined signaling sequences (promoters, terminators, Shine-Dalgarno-like sequences, start and stop codons) allows us to detect the coding sequences. These coding sequences are translated into abstract "proteins" that interact with one another and produce a phenotype that can be more or less welladapted to the environment. 
To model the activity of proteins and the resulting phenotype, we defined a simple "artificial chemistry" (Dittrich et al., 2001) that describes the organism's metabolism in a mathematical language. In our simplified artificial world, we assume that there is an abstract, one-dimensional space of possible metabolic processes (that is, in this model, a metabolic process is just a real number). In this "metabolic space", each protein is involved in a subset of processes which is described using the fuzzy set formalism: a given protein can be involved in a metabolic process with a possibility degree lying between 0 and 1 . A protein is thus fully characterized by a mathematical function that associates a possibility degree to each metabolic process. For simplicity, we use piecewise-linear functions with a symmetric, triangular shape (Fig. 1). In this way, only three numbers are needed to characterize the metabolic activity of a protein: the position $m$ of the triangle on the axis, its half-width $w$ and its height $h$. This means that the protein contributes to the range $] m-w, m+w$ [ of metabolic processes, with a preference for the processes closest to $m$ (for which the highest efficiency, $h$, is reached). Thus, various types of proteins can co-exist, from highly efficient and highly specialized ones (small $w_{+}$high $h$ ) to polyvalent but poorly efficient ones (large $w$, low $h$ ).

In this framework, each coding sequence is translated into a chain of abstract "Amino-Acids" (AA) using an artificial genetic code (shown in Fig. 1). This primary sequence is decomposed into three interlaced binary subsequences that will in turn be interpreted as the values for the $m_{,} w$ and $h$ parameters. For instance, the codon 010 (resp. 011 ) is translated into the single $A A W_{0}$ (resp. $W_{1}$ ), which means that it contributes to the value of $w$ by adding a bit 0 (resp. 1) to its binary code. Thus, small mutations in the coding sequence (substitutions, indels, possibly causing frameshifts) will change these parameters, and hence the metabolic activity of the protein.

In the RAevol model each protein may have a regulatory activity beside its metabolic activity: it can interact with promoter sequences, thus enhancing or inhibiting the transcription of other genes. To determine whether a protein can regulate a particular promoter, we test whether the AA-chain of the protein contains a small motif that can bind to a subsequence of this promoter. The set of motifs that can bind to a particular DNA subsequence is randomly determined once and for all at the beginning of the evolutionary run. Like in most bacteria, the sign of the regulation depends on whether the binding occurs up or downstream from the first transcribed nucleotide (Janga and Collado-Vides, 2007). The resulting transcription level is used to scale up or down both the metabolic activity (height of the triangle) and the regulatory activities of the protein. We call the proteins that actually have a regulation activity Transcription Factors (TFs). Note that proteins with no metabolic activity (null $w$ or $h$ ) can nevertheless be TFs. In this case, they are called pure TFs.

Due to this regulatory process, the transcription levels of the genes (and hence the protein concentration levels) may vary during the lifetime of the organism. At each time $t$, the global metabolism is computed by combining all the protein curves scaled by their concentrations. The phenotype of an artificial organism is thus defined as the dynamic curve showing the degree of realization of each possible metabolic process at each time $t$. The fitness of the organism is then computed on the basis of the distance between the phenotypic curve and a pre-defined target curve (representing the metabolic functions needed to survive in the environment). Fitter organisms are more likely to replicate (see Section 5), with small mutations and large rearrangements (duplications, deletions, inversions, translocations) occurring at random locations during genome replication. Genome size, gene number and gene order are hence free to evolve. Rearrangements can modify the topology of the network (duplication or deletion of genes or promoter regions). Small mutations in coding sequences or in promoters can

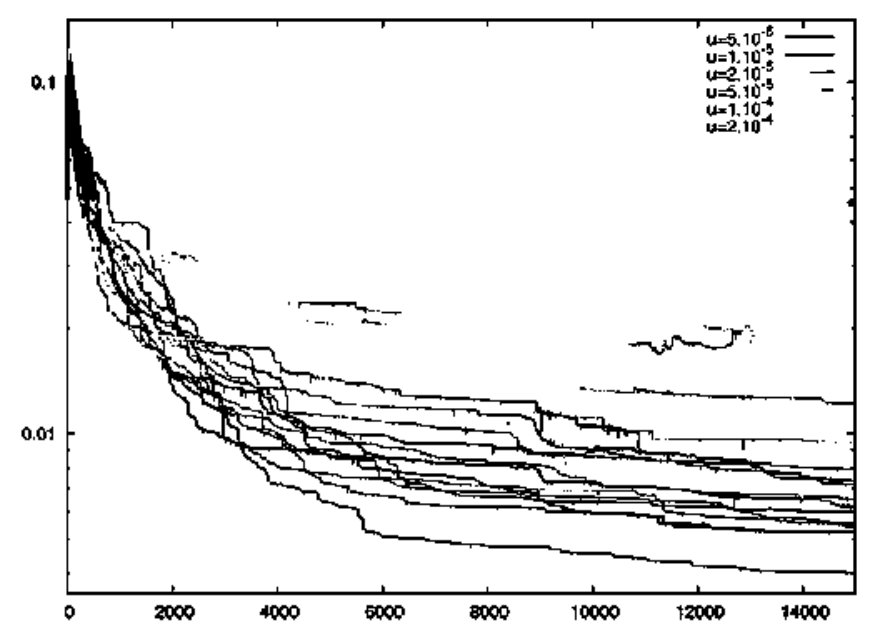

Fig. 2. Evolution of the metabolic error of the best organisms of each simulat ion during 15,000 generations ( $\log$ scale). Whatever the mutation rate (except the highest) all organisms pertorm similatly.

also affect the DNA-protein bindings and hence the wiring of the network.

\section{Results}

The typical use of digital genetics models is quite close to experimental evolution procedures (Elena and Lenski, 2003): populations of organisms are initialized and left to evolve in controlled conditions (i.e., controlled parameters). By observing the products of the evolutionary process in different conditions and by comparing them, we can unravel the direct or indirect pressures that constrain the structure of the organisms.

RAevol makes it possible to evolve digital organisms in demanding environments where they must react to external signals. Eventually, our objective is to use RAevol to understand how regulation networks evolve depending on external conditions and on the complexity of the environment (e.g., number of states, frequency or periodicity of environmental variations...). However, here, we intentionally let all the populations evolve in an identical, steady environment. Indeed, such a null experiment is necessary to test the classical idea that the environmental complexity is a major determinant of the complexity of the network. We have already shown that in Aevol the complexity of the genomes is strongly determined by the mutation rate (Knibbe et al., 2007a). We hence conceived this null experiment to test whether this pressure is strong enough to influence the complexity of the networks too, even in a simple environment.

We let the organisms evolve in a constant environment: 18 different populations of 1000 organisms evolved under 6 different mutation rates $u$ (from $5 \times 10^{-6}$ to $2 \times 10^{-4}-$ defined as the per-nucleotide probability of a small mutation or a rеaгrangement occurring during replication), the selective pressure and the environment being strictly identical for all the experiments.

During the evolutionary process, the organisms progressively acquire new genes and connect them in such a way that they fulfill the task they are selected for (Figs. 2-4). All the simulations proceed qualitatively in a similar way, evolving quickly in the first stage of evolution (rapid gene acquisition) then slowing down the process of gene acquisition while optimizing the sequence of existing genes and promoters. However, looking at the evolution of the size of the genome and the number of genes, we can see a clear trend for lower mutation rates to have larger genomes (Fig. 3) containing more genes (Fig. 4). 


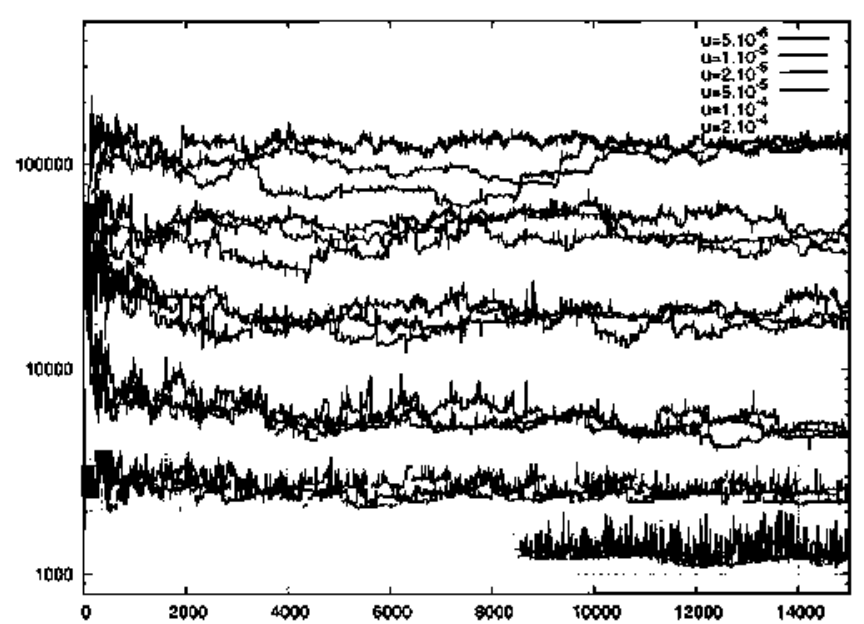

Fig. 3. Evolution of the size of the genomes (in bp, log scale) of the best organisms during 15,000 generations. The size of the genomes appears to be strongly dependent on the mutation rate $u$. Note that, in the model, genome size depends on both the number of genes and the size of non-coding sequences.

We analyzed the structure of both the genomes and the regulation networks of the best organisms after 15,000 generations. We found that all the features of the evolved organisms are influenced by the mutation rate: the organisms are clearly more complex when the mutation rate is low (Figs. 5 and 6 ) even though they all evolved in an identical and steady environment.

These results confirm the ones we had previously obtained with Aevol: the total coding length is influenced by the spontaneous mutation rate and, much more surprisingly, the amount of noncoding sequences is likewise influenced (Fig. 7). With RAevol, we observe that the genetic network scales as well: the size and complexity of the network are clearly correlated with the mutation rate. In the simulations presented here, the environment is steady during the lifetime of the organisms. Thus, there is no direct pressure to evolve a regulatory network at all. Despite this, the lower the mutation rate, the more complex the evolved network. Both the number of genes and the number of TFs are inversely correlated with the mutation rate (Fig. 8). But as the mutation rate decreases, the number of TFs increases faster than the number of genes. This trend is even clearer in our runs if we consider the pure TFs (proteins with a regulatory activity but no contribution to the metabolism, Fig. 9).

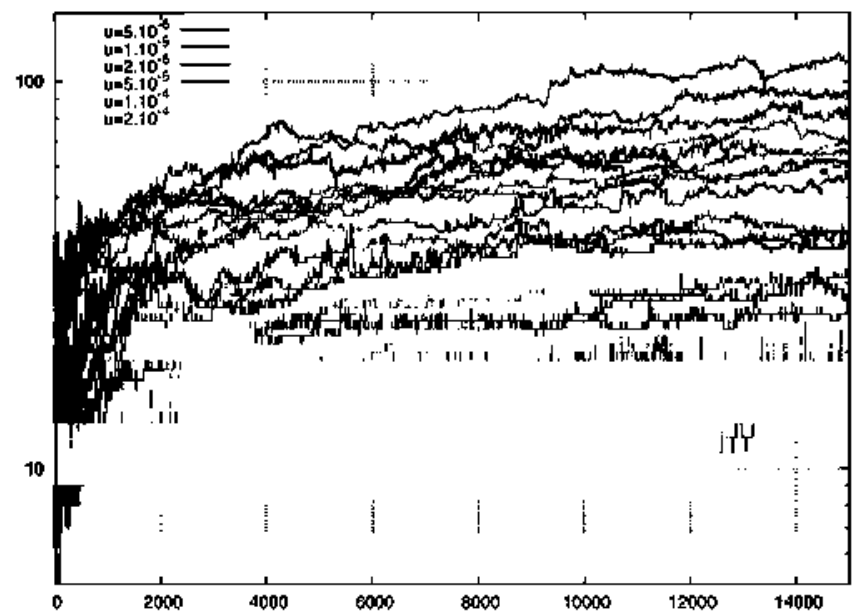

Fig. 4. Evolution of the number of genes of the best organisms ( $\log$ scale) during 15,000 generations. After a short period of fast gene recruitment, the number of genes stabilizes. The number of genes in the organisms appears to be strongly dependent on the mutation rate $u$.

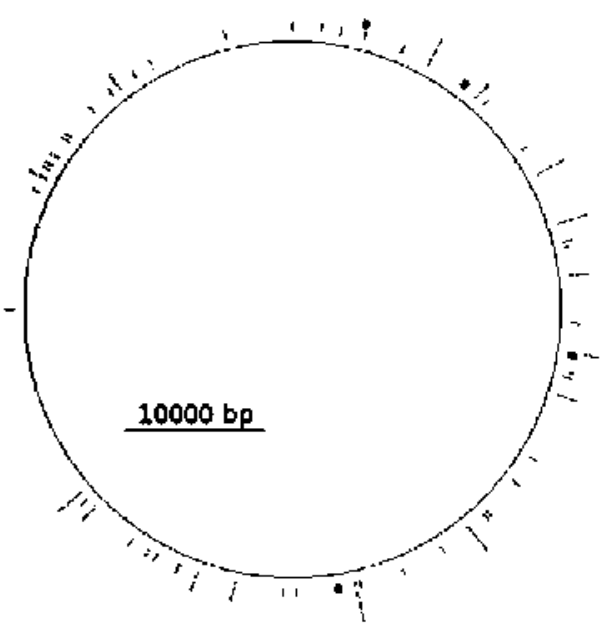

(a)
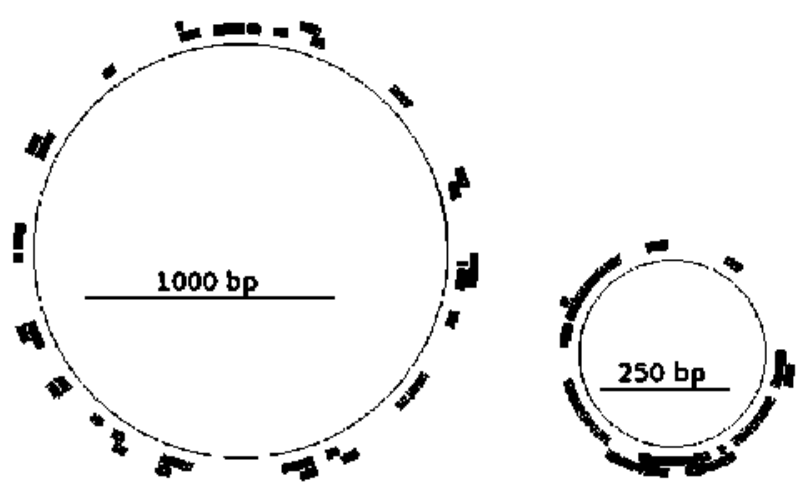

(b)

Fig. 5. After 15,000 generations, the genomes range from large ones (a) to intermediate and small ones (b) depending on the mutation rate $u$. These differences are due to robustness and evolvability constraints: large genomes cannot be maintained when organisms face high rearrangement rates, On the opposite, under low rates, large genomes ate more evolvable (see Knibbe et al., 2007a and Section 4) On each figure the circle represents the whole genome (scale is different on each figure) while the gray arcs represent the coding regions. The color code is arbitrary. similar gray levels representing similar metabolic functions (i.e., proximity in the $\Omega$ space; see Section 5).

\section{Discussion}

As Figs. 10 and 11 show, our experiments with RAevol reproduce qualitatively the scaling laws observed in the prokaryotic kingdom (Cases et al., 2003; van Nimwegen, 2003; Konstantinidis and Tiedje, 2004; Molina and van Nimwegen, 2008). Small genomes with few genes only have a very basic regulation activity while large ones develop complex regulation networks with many genes. Both the number of genes having a metabolic activity and the number of genes having a regulatory activity scale as power-laws of the total gene number, but when the former scales with an exponent below 1 , the latter shows a super-linear scaling.

In our experiments, all organisms evolved in the same - simple - environment. Thus, environmental conditions cannot have caused the scaling of the genetic complexity here. The only difference between our organisms is the mutation rate $u$ that ranged from a very high one $\left(u=2 \times 10^{-4}\right.$ mutations per bp per replication) to a low one ( $u=5 \times 10^{-6}$ mutations per bp per replication). As Figs. 7-9 show, the mutation rate is the crucial factor determining the organisms' complexity. This is what we observed with the Aevol model in which proteins had no regulatory activity. We showed that this scaling was the consequence of an indirect selection of lin- 


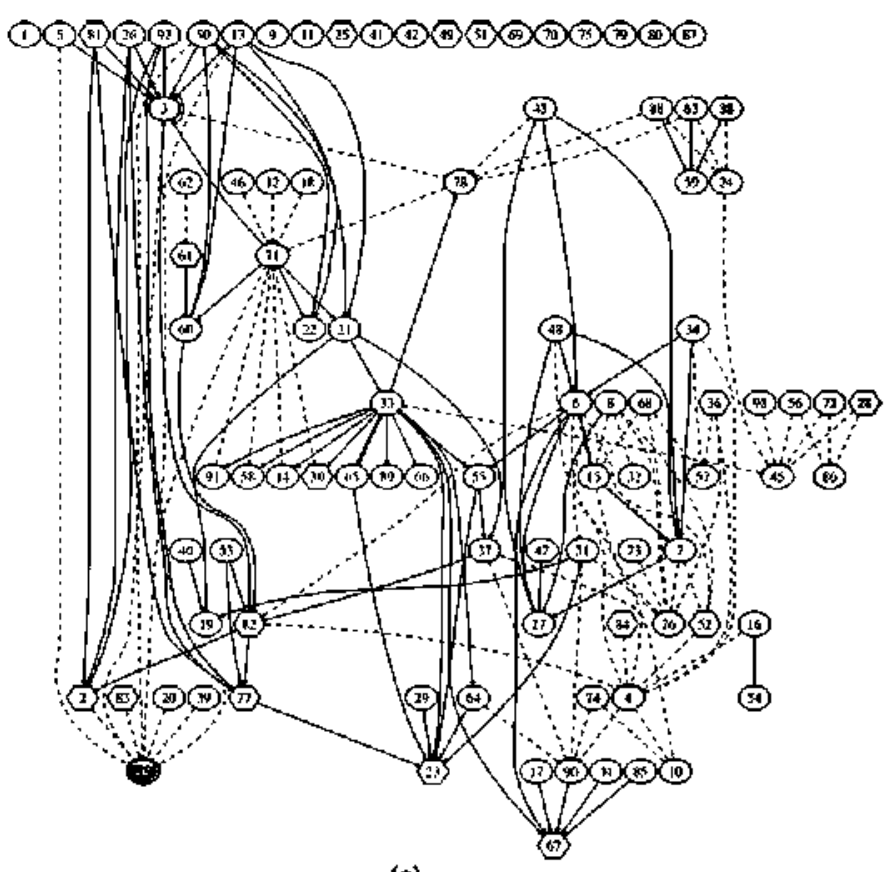

(a)

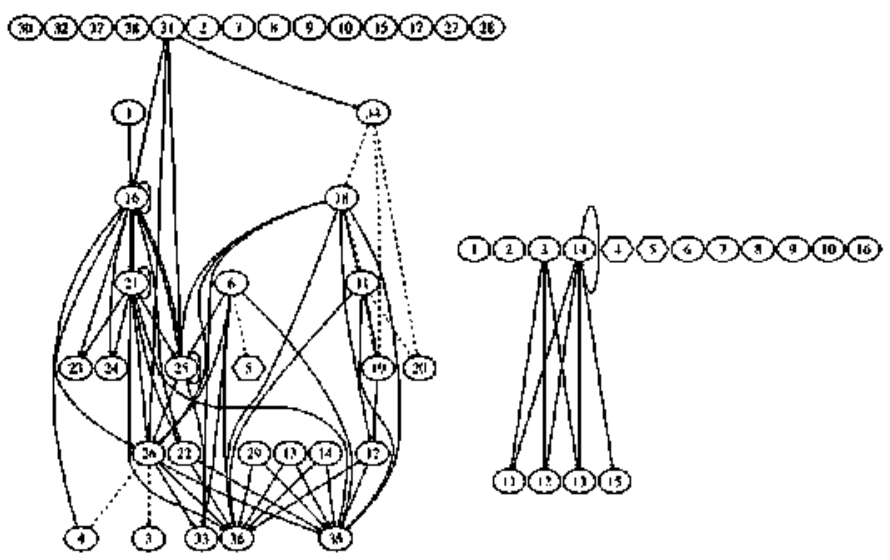

(b)

Fig. 6. After 15,000 generations, the complexity of the gene networks ranges from a high connectivity (a) to medium and low ones (b) depending on the mutation rate 4. Solid lines represent activation links while dashed lines represent negat ive links. Genes having a metabolic activity are represented by ellipses. Hexagons represent genes wit hout any met abolic activity.

eages whose genomic structure allows for an appropriate trade-off between robustness and evolvability (Knibbe et al., 2007a,b, 2008). If the per-base mutation rate is high, large genomes with many genes cannot maintain their fitness due to the mutational load they undergo. Large non-coding sequences cannot be maintained either, because they promote large chromosomal rearrangements that can affect some genes. On the contrary, if the mutation rate is low, large genomes can maintain themselves in the population and they can even outcompete the smaller ones, because they can fit the target more precisely with more genes, and because they are more likely to find a beneficial mutation. We showed for Aevol that this trade-off between robustness and evolvability manifested itself by the survival of the lineages whose expected fraction of neutral offspring $F_{v}$ (the expected fraction of offspring without mutation or only neutral ones at each reproduction) was close to $1 / W$. where $W$ is the number of reproductive trials of the best individual (Knibbe

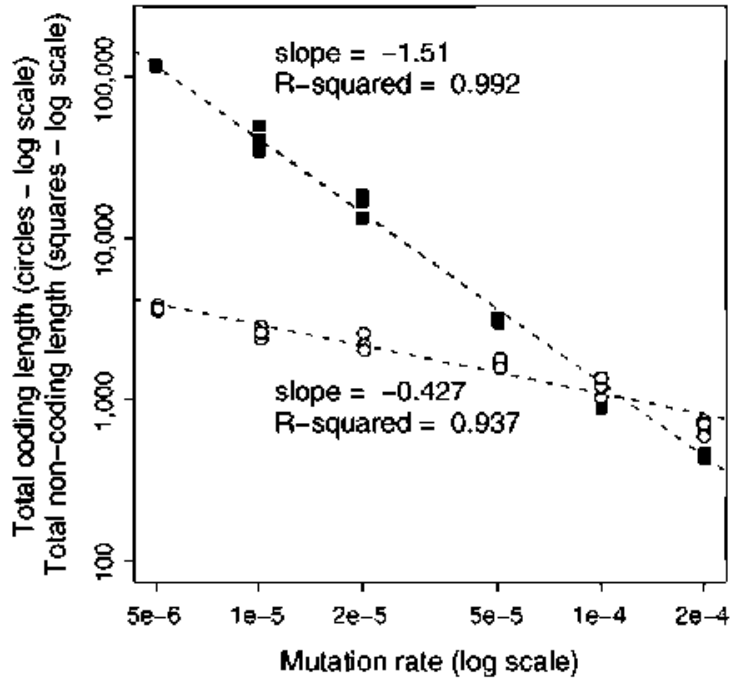

Fig. 7. Size of the coding (gray circles) and non-coding (black squares) sequences for the best organisms of the 18 simulations at generation 15,000 ( $\log$ - $\log$ plot) Both values cleatly scale with the mutation cate

et al., 2007a). In the experiments presented here, the evolved $F_{v}$ is again close to $1 / W$ in most runs (Fig. 12). This suggests that the present results can also be explained by indirect pressures on the global mutational variability of the genome.

All the scaling laws observed in RAevol can derive from this pressure for robustness and from the scaling it imposes on the number of genes. Indeed, as the number of genes increases, the number of promoters also grows (possibly a little slower because of operon structures). Thus, the number of putative regulatory genepromoter associations grows quadratically. Since, in the model, the regulatory activity is computed through a combinatorial algorithm that associates protein primary sequences with promoter sequences (see Section 5), a linear increase in the number of promoters leads, for a protein with a regulatory motif, to a linear increase in the number of potential targets in the genome. As a consequence, a protein owning a regulatory motif has a higher probability of being a TF (number of actual targets in the genome greater or equal to 1 ) in a large genome than in a smaller one.

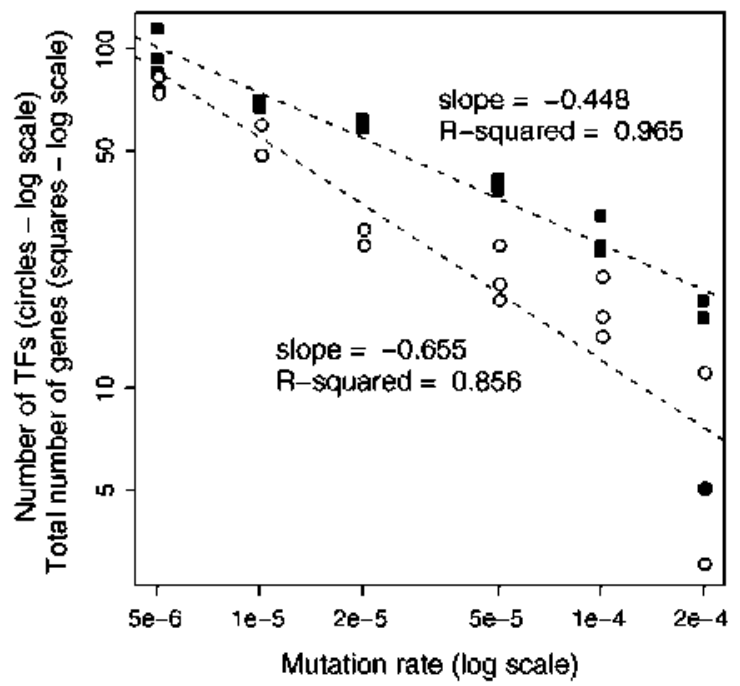

Fig. 8. Total number of genes (black squares) and number of Transcription Factors (gray circles) for the best organisms of the 18 simulations at generation 15,000 $(\log -\log$ plot $)$. Both values clearly scale with the mutation rate but the number of TFs grows faster than the number of genes. 


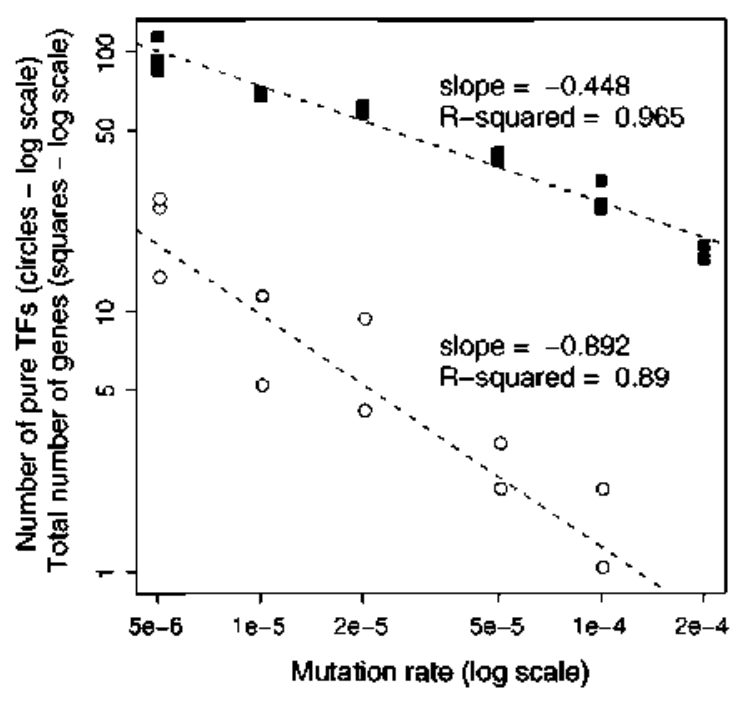

Fig. 9. Total number of genes (black squares) and number of pure TFs (gray circles: Pure TFs ate proteins having a regulation activity but no metabolic contribution) for the best organisms of the 18 simulations at generation 15,000 (log-log plot). Both values clearly scale with the mutation rate but the number of pure TFs grows faster than the number of genes.

Thus, RAevol appears as a null model in which links in the networks are added with an almost constant probability when the number of gene-promoter pairs increases. Consequently, in the model, the scaling of the number of genes (due to mutational robustness constraints) leads naturally to a super-linear increase in the number of regulatory nodes.

Whether a similar mechanism can explain the quadratic growth of Transcription Factors observed by van Nimwegen (2003) and Molina and van Nimwegen (2008) is an open question. Since real transcription factors have one or more DNA-binding domains that are well defined units on the structural, functional and evolutionary level, it is not clear whether such combinatorial process is at work in real genomes. Yet, several authors have reported the combinatorial properties of the binding between TFs and their DNA targets. According to Itzkovitz et al. (2006), the number of degrees of free-

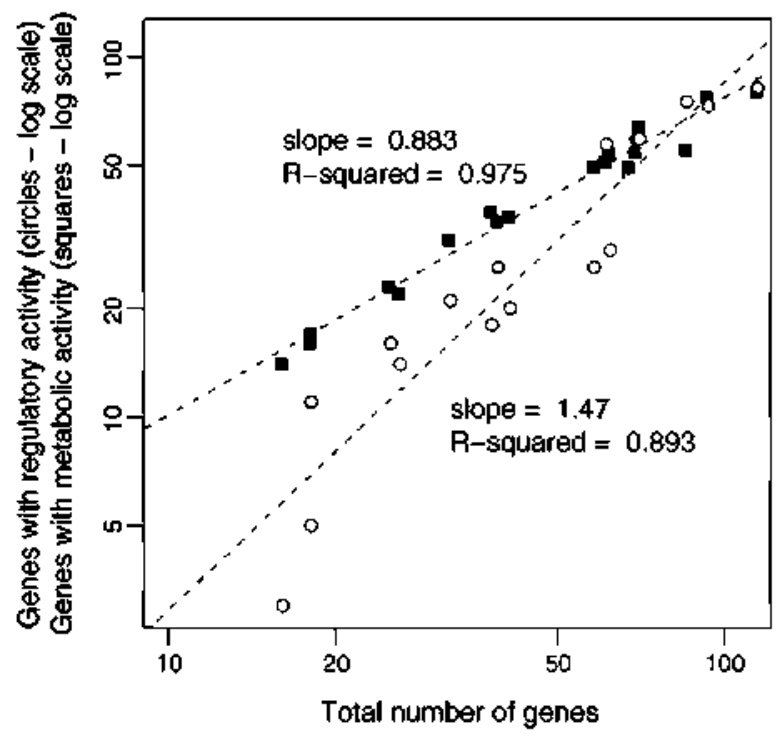

Fig. 10. Number of genes involved in metabolism (black squares) and in the regulation process ( $g$ ray circles) as a function of the total number of genes in the genome (best organisms of the 18 simulations at generation 15,$000 ; \log -\log$ plot). Dashed lines show power-law fits.

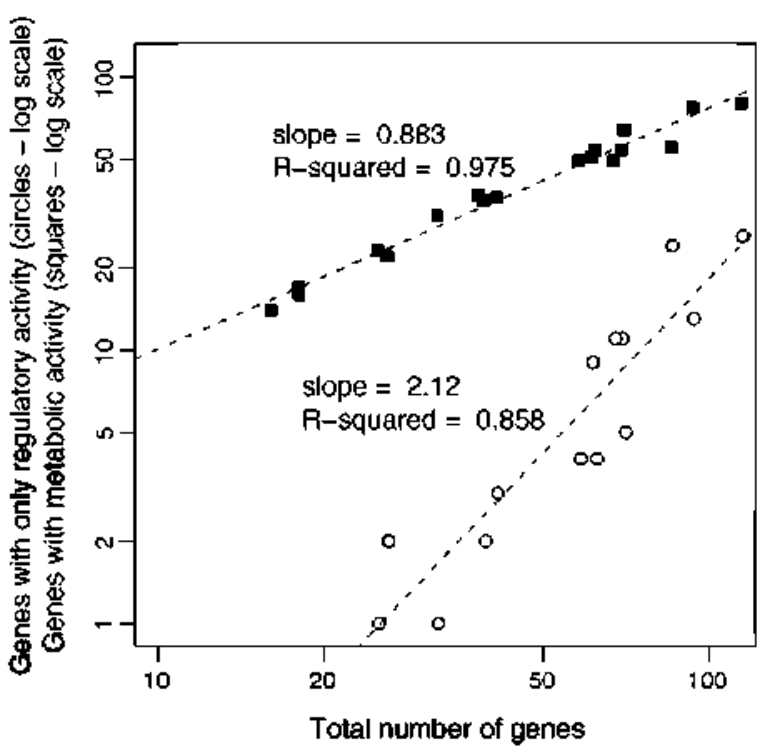

Fig. 11. Number of genes involved in metabolism (black squares) and number of pure TFs (gray circles) as a function of the total number of genes in the genome (best organisms of the 18 simulations at generation 15,000; $\log -\log$ plot). Dashed lines show power-law fits.

dom of the binding mechanism can partly account for the increase in the number of TFs. Moreover, it is also known that TFs can bind to a broad spectrum of binding sites with different affinities and change targets widely among species (Balleza et al., 2009).

Maybe the most striking result of our simulations is that the super-linear growth of the number of TFs is also observed for pure TFs. Moreover, these proteins scale more than quadratically with the number of genes (Fig. 11). One can propose different hypotheses to explain the appearance and fixation of pure TFs. They can appear due to random mutations but they most likely result from duplication/divergence events (e.g., gene copies that lose their metabolic activity while retaining their regulation activity). The interesting question is why evolution maintains such genes in the simple environment where our organisms live. One can assume that, when the number of genes increases, there is a need for more regulation

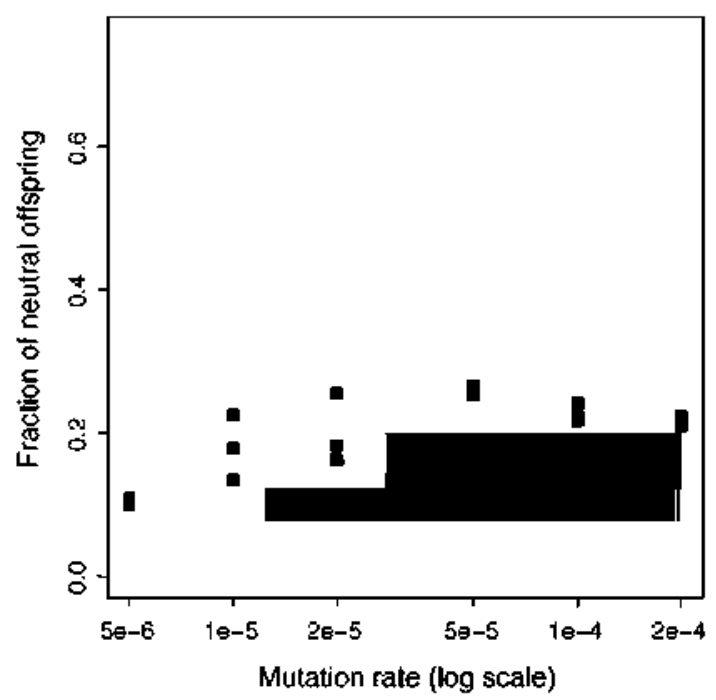

Fig. 12. Evolved tract ion $F_{\mathrm{r}}$ of neutral oftspting. $F_{\mathrm{r}}$ was estimated by torcing the final best organism of each nin to reproduce itself 10,000 times (with the same mutation rate as during the run) and by counting the number of offspring that have the same fitness as their progenitor. The gray area represents organisms whose fraction of neutral offspring is lower than $1 / W$. 
in order to position the attractor of the network more precisely in a space in which the number of dimensions increases. In this hypothesis, pure TFs could be directly selected. Alternatively, one can suppose that they are indirectly selected, but their contribution to the robustness/evolvability balance is very difficult to assess. They can contribute to the organism's robustness if they have a canalizing effect. Pure TFs can also contribute to the organism's evolvability by enabling small mutational variations that are more likely to be positive than mutations in metabolic genes. In this hypothesis, pure TFs would be conserved because their mutation can finely tune the activity of their target proteins without changjng the metabolic processes these targets are involved in. We now plan to analyze the phylogeny of our organisms to study specifically the mechanisms that lead to the appearance and to the fixation of these "pure" regulators. We also plan to use KnockOut experiments to better understand their contribution to the dynamics of the regulation network.

To conclude, our results show that, at least in our model, the scaling laws reflect fundamental principles of bacterial evolution, i.e. the selection for an appropriate balance between robustness and evolvability (Lenski et al., 2006). Our simulations show that the pressure for complexification of the network can be indirect, unrelated to differences in the environment or the lifestyle: when facing identical environmental constraints, the organisms' structure can range from very simple life forms (with a reduced gene set and loose connectivity) to very complex ones, the main determinant of the structure being "only" the mutation rate here. of course, this does not imply that, if faced with an environment of variable complexity and demand, organisms with the same mutation rate will have a similar structure. However, we can deduce from our results that the molecular complexity of the organism will be bound by the robustness constraint, meaning that the mutation rate will still be a major factor in determining organismal complexity.

\section{Methods}

\subsection{Population inifdalization}

Each population is seeded with 1000 asexual individuals with an identical genome. This initial genome is a random binary sequence of 5000 base pairs (bp) contaiming at least one coding sequence. Each run is seeded with a different initial genome.

\subsection{Detection of transcribed regions}

The transcription algorit lim seatches tor promoters on each strand. Then, for each ptomoter, it follows the strand until it finds a terminator. This delimits the transcribed region. Note that several promoters can share the same terminator. In this case transc ribed regions overlap.

Promoters are sequences similar to a pre-defined consensus. In the experiments presented here, the consensus sequence was 0101011001110010010110 and $d \leq d_{\max }=4$ mismatches wete allowed. Terminators are sequences able to torm a stem-loop st ructure, as the $\rho$-independent bacterial terminators do (here the stem size was set to 4 and the loop size to 3). We assign a ground expression level $\theta$ to the transc ribed region depending on the similarity of the promoter with the consensus (Struhl, 1999): $\beta=1-\left(d /\left(d_{\max }+1\right)\right)$.

\subsection{Detection of coding seglences and transiation process}

Once all transcribed regions have been localized, they are parsed to detect the initiation and termination signals of translation. These signals delimit the coding sequences. The initiation signal is the motif $011011^{* * *} 000$ (Shime-Dalgarnolike signal followed by a start codon, 000 here). The termination signal is the next stop codon $(001)$ on the same reading trame. Each time an initiation signal is fou nd. the following positions are read three by three (codon by codon) until a stop codon is encountered. A transcribed region can contain several coding sequences (overlapping or not), meaning that operons are allowed.

Eacli coding sequence found inside a transcribed tegion is read triplet by triplet (codon by codon) and an attificial genetic code is used to translate it into a chain of artificial amino-acids. In this genetic code (shown in Fig. 1), there are 6 different amino-acids, grouped into thee pairs $\left(M_{0} / M_{1}, H_{0} / H_{1}\right.$ and $\left.W_{0} / W_{1}\right)$.

\subsection{Metabolic activity of proteins}

Let $\Omega$ be the abstract space of metabolic processes. To keep the model simple. $\Omega$ is one-dimensional space, more precisely a real interval: $\Omega=\mid a, b]=\mathbb{R}$ (with $0=0$ and $b=1$ in the experiments presented here). Eacl protein i can contribute to (or inhibit) a fuzzy subset of $\Omega$. This tuzzy subset is fully characterized by a mat hematical function $\left.f_{\mathrm{S}}: S=\mid a, b\right] \rightarrow[0,1 \mid$. This function is called a possibility distribution. It defines, for each metabolic process $x$ the degree of possibility $f_{5}(x)$ with which the protein $i$ can perform the process $x$. A metabolic process $x$ belongs to the fuzzy set of a protein if $f_{i}(x)>0$. We use piecewise-linear distributions with a symmetric triangulat shape. Sucli distributions can be characterized by thee parameters: the position m (mean) of the triangle on the axis, its lieight $h$ and its lialt-width $w$. Hence a protein i can be involved in the metabolic processes ranging from $m_{i}-w_{1}$ to $m_{i}+w_{i}$, with a maximal degree of possibility for the process $m_{i}$. The function of the protein is thus non-null on the interval $] m_{i}-w_{i} . m_{i}+w_{i} \mid$.

In computational terms, the amino-acid chain of a protein is interpreted as three interlaced variable-length binary codes, giving the values of $m_{i}, w_{1}$ and $h_{i}$ respectively. To compute the value of $m_{1}$ tor example, we extract all $M_{0}$ and $M_{1}$ amino-acids fou ind in the chain. They will form the Gray encoding of m (the Gray code is a binary numeral system where two successive values ditter in only one bit). It the first $M$ amino-acid of the chain is a $M_{0}$ (resp. a $M_{1}$ ), then the first bit of the Giray code of $m_{\mathrm{f}}$ is a 0 (resp. a 1 ), and so on. Thus, if the chain contains $n$ amino-acids of type $M$, we get a Gray code of size $n$, which encodes an integer between 0 and $2^{n-1}$. A normalization enables us to bring the value of the parameter into the allowed range, that is, $[a, b]$ tor $m$. The same method is used to compute the values of $w_{1}$ and $h_{1}\left(-1 \leq h_{1} \leq 1\right.$ and $0 \leq w_{1} \leq w_{\max }, w_{\max }=0.03$ hete). If $h_{i}$ is positive, the protein cont ributes to the metabolic processes. If $h_{i}$ is negative, it impedes these processes. If $h_{i}$ or $w_{i}$ equals 0 it has no metabolic activity.

\section{Regulatoly activity of proteins}

In RAevol, the transcription rate of a protein may vary throughout the lifetime of the artificial organism. It depends both on the intrinsic activity of the promoter (ground level, see above) and on the regulatory activity of the other proteins. Thus the concentration of a protein $l$ is a function of time $c_{p}(t)$. This concentration is used to scale up or down the metabolic activity of the proteint the int rinsic distribut ion described above (triangle centered on $m_{5}$, of lialt-widtl $w_{1}$ and of height $\left.h_{i}\right)$ is multiplied by $c_{i}(t)$ at each time step. These scaled possibility distributions are those used to compute the phenotype at each time step (see below). This reflects the fact that a very efficient protein (high $h_{i}$ ) has actually no effect when it is not expressed. Similarly, the current concentration $c_{1}(t)$ of a protein also scales up or down the regulatory influence of the protein $i$ on the other proteins at time $t$.

The possibility that a given protein will bind to a specitic ptomoter is determined by a "value of affinity" between the amino-acid chain of the former and the genetic sequence of the latter. Small amino-acid motifs, which will henceforth be referred to as regulation domains, are able to bind to specific DNA subsequences with a given affinity. If a protein contains several regulation domains, its global attinity value over the promoter will be given by the best one among them. This value of afionity is used to determine the strengtl of the protein's influence on the transcriptional activity of the promoter it binds to. Like in most bacterial promoters, the nature of the regulation (activat ion or inhibition) depends on whether the binding occurs before (upstream) or after (downstream) the position of the first transcribed nucleotide (Janga and (ollado-Vides, 2007). Thus, in RAevol, a promoter is composed of thiee DNA subsequences; the consensus sequence (where the RNA polymerase starts the transcription process) and its two flanking regions. When bound upstream, a protein enhances the transcriptional activity and, on the opposite, when bound downstream, it represses the activity of the polymerase, thus reducing the transcriptional activity.

The sequences that are able to interact with a specific DNA subsequence (thus constituting the possible regulation domains) are randomly determined at the beginning of the evolut ionaty cun. In RAevol, regulation domains are small 5-AminoAcid (AA) sequences that may have all affinity with 20-bP DNA sequences. To compute this aftinity value, we align the regulation domain with the DNA sequence and compute the local affinity of each AA with the 4-bp subsequence it faces (Fig. 13). The motif will be able to bind the DNA sequence only if all five AA have strictly positive affinities with their corresponding DNA subsequences.

$A$ binding matrix $B$ is defined which contains the affinity of each amino-acid witheach 4-bp sequence. Given our artiticial chem istry principles, we have 7 possible amino-acids (Statt, $M_{0}, M_{1}, H_{0}, H_{1}, W_{0}$ and $W_{1}$ ) and $2^{4}=164$-bp sequences. Tlius, $B$ is a 7,16 matrix. Bycloosing the initialization procedure of the regulatory matrix, we are able to choose the probability for a given motif to have a regulat ion activity. In all the experiments presented here, $B$ was randomly initialized (uniform distribution in [0,1]) and subsequently filled with $75 \%$ of null values. Thus, the probability that a given motif will bind to a specific DNA sequence of 20 -bases long (length of the regulation sites in RAevol) is less than $0.1 \%$. As a consequence, the probability that a 20-AA-long protein will be able to up-regulate (resp. down-regulate) a given promoter can be estimated at around $5 \%$ (probability to contain a motif that binds the promoter of the regulated gene). 


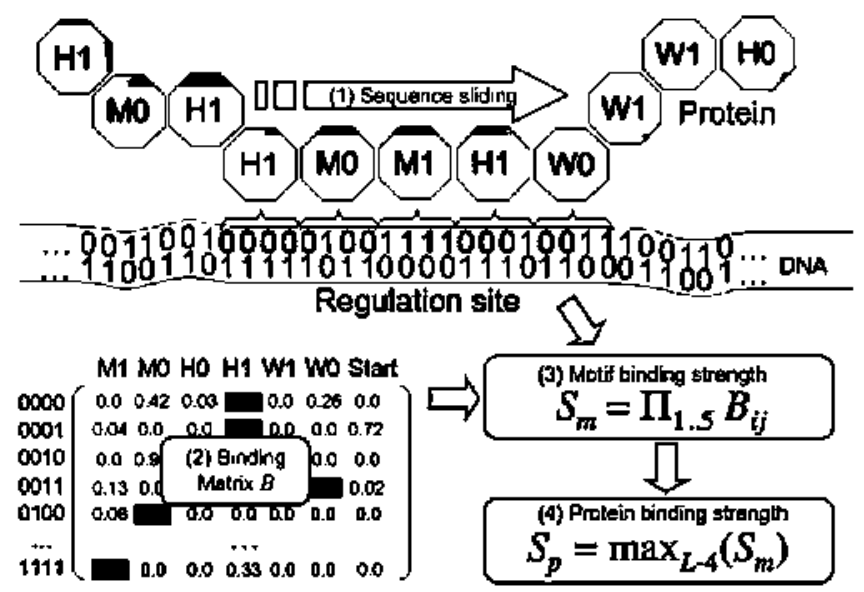

Fig. 13. Computation of the binding between TFs and regulation sites. (1) The protein primary sequence slides in front of the 20-bp regulat ion site and all5-AA motifs are tested. (2) For each AA-subsequence pair, the binding value $B_{\|}$is read in a Binding Matrix $B$ (see text for the initialization of the binding matrix). (3) The binding strengtl of the whole motif is the product of the five $B_{y}$ values and (4) the binding strength of the whole protein is the maximum strength over the $L-4$ motifs it cont ains ( $L$ being the length of the proteins primary sequence).

The activity of a promoter depends on the sum of the activities of activators $\left(A_{1}(t)=\sum c_{1}(t) A_{y_{1}}\right)$ and on the sum of the activities of the inhibitors $\left(h_{1}(t)=\sum g_{f}(t) J_{\mu}\right)$, where $A_{11}$ (resp. $I_{11}$ ) is the affinity of protein $j$ on the enhancer of the promoter $i$ (tesp. on its operator) and $c_{j}(t)$ is the concentration of protein $j$ at time $t$. When $A_{\mathrm{I}}=\mathrm{I}_{\mathrm{I}}=0$ (no regulation), the promoter lias a ground activity $\beta_{i}$ (Strulil, 1999). If $A_{5}>0$ this activity increases progressively up to a maximum level, If $t_{i}>0$, it decreases progressively to zero. The transcription rate $e_{i}$ over time is then given by Hill-like functions:

$e_{1}(t)=\beta_{i}\left(\frac{\theta^{\mathrm{n}}}{J_{i}(t)^{\mathrm{n}}+\theta^{n}}\right)\left(1+\left(\frac{1}{\beta_{i}}-1\right)\left(\frac{A_{i}(t)^{\mathrm{n}}}{A_{i}(t)^{\mathrm{n}}+\theta^{n}}\right)\right)$

where nand $\theta$ ate constant coeflicients that determine the sliape of the Hill-function In the simulations presented liere, $n=4$ and $\theta=0.5$. Finally, given the transcription rate, one can compute the protein concentration (tor the sake of simplicity. we assume here that the protein concentration is linearly proportional to the RNA concentration) through a synthesis-degradation rule (Eq. (2)). Thus, when a protein is regulated, its concentration is scaled up or down depending on its transcription rate.

$\frac{\partial c_{i}}{\partial t}=e_{i}(t)-\phi c_{i}(t)$

where $\phi$ is a temporal scaling constant representing the protein degradation rate At each time step, the regulatory activity e of all proteins is computed depending on theif concentration and binding affinity. Then, the concent rations are updated according to Eq. (2) on the basis of a simple synchronous Euler integration scheme.

The transcription regulation in RAevol is a simplification of the teal mechanisms of DNA-protein interaction. However, it catches the main mechanisms of genetic regulation while remaining computationally tractable. It also allows for proteins that perform a metabolic activity without any regulatory act ivity or, on the opposite. for proteins without any metabolic activity (i.e., $\int_{0}^{1}|f(x)|=0$ ) to have a regulatory activity. We call "Transcription Factors" (TFs) the proteins that have a tegulatory activity ( regardless of their metabolic activity). Proteins having a regulation activity without contributing to the metabol ism are called pure Transcription Factors.

\subsection{Phenotype computation}

Once all the proteins encoded on the genotype of the organis m lave been identified, the global phe notype can be computed by combining the whole set of proteins. We use the same formalism for the phenotype as for the proteins: the phenotype is the fuzzy subset of metabolic processes that the organism is able to perform. This fuzzy subset is described by a possibility dist ribution P ind icating to what extent the organism is able to pertorm each process of $\Omega$. The fuzzy logic fiamework provides us with logical operators to compute the complement, the union and the intersection of tuzzy subsets. Hete, in logic terms, the global tunctional abilities of an individual are the metabolic processes that are enabled AND NOT disabled by the proteins of the organism.

$P=\left(\cup_{i}\left(f_{i} \mid h_{1}>0\right)\right) \cap \overline{\left(\cup,\left(f_{i} \mid h_{3}<0\right)\right)}$

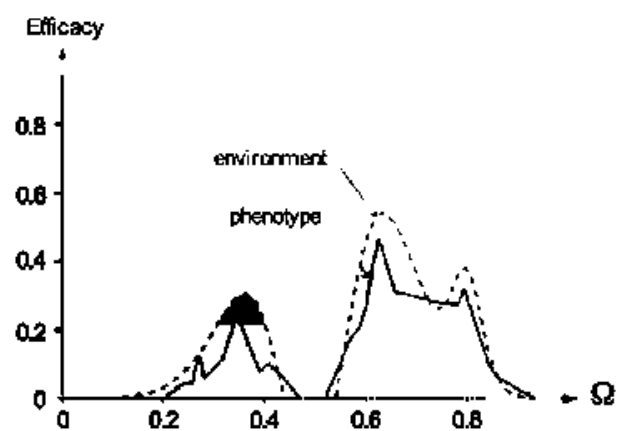

Fig. 14. Measure of an individual adaptation, Dashed curve: envitonmental dist ribution $E$. Solid curve: pltenotypic distribution $P$ (resulting metabolic protile obtained after combining all proteins). Filled area: metabolic error $g$.

Here, we use Lukasiewic $z^{*}$ fuzzy operators. For two proteins characterized by the distributions $f_{1}$ and $f_{2}$ respectively, Lukasiewicz operations are defined as tollows:

$$
\begin{cases}\text { NOT } & f_{\text {now } 11}(x)=1-f_{1}(x) \\ \text { OR } & f_{1 \cup 2}(x)=\min \left(f_{1}(x)+f_{2}(x), 1\right) \\ \text { AND } & f_{102}(x)=\max \left(f_{1}(x)+f_{2}(x)-1\right.\end{cases}
$$

\subsection{Fitmess evaluation}

Using our attificial chemistry, we are able to map a genotype to phe notype, the latter being a dynamic function $P(t)$ which expresses the metabolism of the organism in the abstract functional space $\Omega$. This enables us to evaluate each organism and to compute its "metabolic error" $g$ in a given environment: the environment is described as a target (fuzzy) set of metabolic processes that lave to be tulfilled by the cell in order to be able to reproduce. The metabolic error is computed as the atea of the gap between both functions (Fig. 14). The lower the metabolic error, the higher the reproduction probability.

Since the phenotype is a dynamic function, the environment may also be a dynamic function $E(t)$. Depending on the experiment one wants to do with the model, the metabolic error can be computed only once (e.g., after a transient period). at regular steps, during a time interval or after a particular environmental event. In this last case, the event can be sensed by the cell thiough "signaling molecule" which concentration may follow the environment variation. Hete, the plienotype is computed during 20 time steps, the gap being computed at each time step during the second half. The metabolic error is then the mean of the 10 gap values. As a consequence, we positively select for the networks that reach a steady state.

\subsection{Reproduction, mutations and rearrangements}

In the cutrent version of RAevol, the population size is constant ( $N=1000$ individuals liete) and the population is completely rentewed at each generation. At each generation, each individual is evaluated and a select ion process is used to determine the number of offspring it will have. Then, all the selected organisms reproduce to create the next generation.

We use the "exponential ranking" selection scheme. At each generation, the individuals are sonted by dec reasing metabolic error, such that the best individual has rank $N$. Then the probability of reproduction of the individual with rank $r$ is $\left.((s-1)\}\left(s^{N}-1\right)\right) s^{N-r}$, whete $\left.s=\mid 0,1\right)$ tunes the intensity of the selection $(s=0.995$ here). Finally, the actual numbers of teptoductions are drawn by a multinomial drawing.

During their replication genomes can undergo seven different kinds of mutations, the first three being point mutations (switches and 1-6 bases indels) and the tour others, large chromosomal rearrangements:

- Translocation: a randomly chosen segment of the genome is moved from its current position to a randomly chosen position.

- Inversion: a randomly chosen segment is inverted from one strand to the other and from one ditection to the opposite one.

- Duplication: a randomly chosen segment is duplicated and reinserted at a randomly chosen position.

- Deletion: a randomly chosen segment is deleted

Mutations affect the genome but can be neutral, for instance when they happen inside non-transcribed, non-coding regions. They can change the size of the genome, the number of genes or the tunctions of the proteins. Indirectly, they can modity the topology of the regulatory metwork, by either duplicating/deleting genes or promoter regions. Findlly, they can modify the aftinities between transcription factors and regulatory regions by changing either the promoter sequences or the regulation domains in the proteins* primary sequences. 
The rate at which mutations occur, u (probability of mutation pet-base pair). is a parameter of the model. Here, in a given rull, $u$ was the same tor all types of mutations. Six rates were tested: $u=5,10^{-6}, 10^{-5}, 2,10^{-5}, 5 \backslash 10^{-5}, 10^{-4}$ and $2,10^{-4}$ per-base pair. For each value, 3 independent runs were carried out.

\section{Acknowledgments}

The authors would like to thank Michael Parsons and JeanBaptiste Rouquier for their help on the manuscript. The BSMC group provides us with the computing resources and we would like to warmly thank Fabien Chaudier for his invaluable help. This work has been funded by the French ANR MDCO Bingo2 2008-2010 project and the Spanish Ministry of Education (project number TIN2007-67148).

\section{References}

Adami, C., 2006. Digital genetics: unravelling the genetic basis of evolution. Nature Reviews Gentics 7 (2), 109-118.

Balleza, E., López-Bojorquez, L.N., Martnez-Antonio, A., Resendis-Antonio, $O$. Lozada-Chávez, I., Balderas-Mattnez, Y.I., Encarnación, S., Collado-Vides, J., 2009. Regulation by transcription factors in bacteria; beyond descript ion. FEMS Microbiology Reviews 33 (1), 133-151.

Cases, I., de Lotenzo, V., Ouzounis, C.A., 2003. Transcription regulation and environmental adaptation in bacteria. Trends in Microbiology 11 (6), 248-253

Cordero, 0.X. Hogeweg. P., 2007. Large changes in regulome size lie rald the main prokaryotic lineages. Trends in Genet ics $23(10), 488-493$.

Dittrich, P., Ziegler, J., Banzhaf, W., 2001. Artificial chemistries-a review. Artificial Lite 7 (3), 225-275.

Drake, J.W., 1991. A constant rate of spontaneous mutation in DNA-based microbes. Proceedings of the National Academy of Science of the USA 88 (16), 7160-7164.

Diake, J.W., Cliatleswortl, B., Cliatleswoth, D., Crow, J.F, 1998, Rates of spontaneous mutation. Genetics 148 (4), 1667-1686.

Elena, S.F, Lenski, R,E., 2003. Evolution experiments witl miccoorganisms: the dynamics and genetic bases of adaptation. Nature Reviews Cienetics 4 (6). $457-469$.

Foster, D.V, Kauffimall, S,A, Socolat, J.E.S., 2006. Network growt l models and genetic regulatory networks. Physical Review E 73 (3 Pt 1), 031912.

Itzkovitz, S. Tlusty. T., Alon, U., 2006. Coding limits on the number of transcription factors. BMC Genomics 7, 239

Janga, S.C., Collado-Vides, J., 2007. Structure and evolution of gene regulatory networks in microbial genomes. Research in Microbiology 158 (10), 787-794.

Knibbe, C., Coulon, A., Mazet, O., Fayard, J.-M., Beslon, G., 2007a. A long-term evolutionaty pressure on the amount of noncoding DNA. Moleculat Biology and Evolution $24(10), 2344-2353$.

Knibbe, $C .$, Fayard, J.-M., Beslon, G., 2008. The topology of the protein network influences the dymamics of gene order: from systems biology to a systemic understanding of evolution. Artificial Life 14(1), 149-156.

Knibbe, G, Mazet, O., Claudier, F., Fayard, J.-M., Beslon, G., 2007b. Evolutionaty coupling between the deleteriousness of gene mutations and the amount of non-coding sequences. Journal of Theoretical Biology 244 (4), 621-630.
Konstantinidis, KT. Tiedje, J.M., 2004. Trends between gene content and genome size in prokaryotic species with larger genomes. Proceedings of the National Academy of Science of the USA 101 (9), 3160-3165.

Koonin, E.V., Wolt, Y.I, Karev, G.P., 2002. The st ructure of the protein universe and genome evolution. Nature 420 (6912), 218-223.

Lenski, R.E., Barrick, J.E., Otria, C., 2006. Balancing robust ness and evolvability. PLoS Biology 4 (12), e428.

Luscombe, N,M., Oian, J., Zliang, Z. Johnson, T., Gerstein, M., 2002. The dominance of the population by a selected tew: power-law behaviout applies to a wide variety of genomic properties. Genome Biology 3 (8), RESEARCH0040.

Maslov, S. Krislina, $S_{*}$ Pang, T. Sireppen, K., 2009. Toolbox model of evolution of prokaryotic metabolic networks and their regulation. Proceedings of the National Academy of Science of the USA 106 (24), 9743-9748.

Molima, N., van Nimwegen, E., 2008. The evolution of domain-content in bacterial genomes. Biology Direct 3,51

Molima, N., van Nimwegen, E., 2009. Scaling laws in tunctional genome content ac ross prokaryotic clades and lifestyles. Trends in Genetics 25 (6), 243-247.

Nehaniv, C.L., 2005. Self-replication, evolvability and asynchronicity in stochastic worlds. In: SAGA, pp. 126-169, corrected version: http:/fhomepages, feis herts.ac.ukf-vcomqcln/nehaniv-SAGA05-withcorrections.pdf.

Pepper, J.W., 2003. The evolution of evolvability in genetic linkage patterns. Biosystems $69(2-3), 115-126$.

Reymond, N., Calevro, F., Vin̄uelas, J., Morin, N., Rahbé, Y., Febvay, C.., Laugier, C., Douglas, A, Fayard, J.-M., Cliatles, $H_{\text {., }}$ 2006. Difterent levels of transcriptional regulat ion due to trophic constraints in the reduced genome of Buchnera aphidicola aps. Applied and Environmental Microbiology 72 (12). $7760-7766$.

Sniegowski, P., Cierrish, P., Johnson, T., Shaver, A., 2000. The evolution of mutation rates: separating causes from consequences. Bioessays 22, 1057-1066

Stover, CK., Pham, X,O. Erwin, A,L., Mizoguchi, S.D., Watrener, P., Hickey, M.I., Brinkman, F.S., Hufnagle, W.O., Kowalik, D.J., Lagrou, M., Gaber, R.L., Cioltry, L. Tolentino, E., Westbrock-Wadman, S., Yuan, Y., Brody, L.L., Coulter, S.N., Folget, K.R., Kas, A, Latbig, K., Lim, R, Smith, K, Spencer, D. Wong, G.K., Wu, Z, Paulsen, I.T., Reizer, J., Saier, M.H., Hancock, R.E., Lory, S., Olson, M.V., 2000 . Complete genome sequence of Pseudomonas aeruginosa pa01, all op portunist ic pathogen Nature 406 (6799), 959-964.

Struhl, K., 1999. Fundamentally different logic of gene regulation in eukaryotes and piokatyotes. Cell 98 (1), 1-4.

van Nimwegen, E., 2003. Scaling laws in the functional content of genomes. Trends in Genetics $19(9)$, 479-484.

Viñuelas, J., Calevro, F., Remond, D., Bernillon, J., Rahbé, Y., Febvay, G., Fayard, J.M., Cliatles, H., 2007. Conservation of the links between gene transcription and chromosomal organization in the highly reduced genome of Buchmera aphidicola. BMC Gienomics 8, 143.

Wagner, G.P., Altenberg, N., 1996. Complex adaptations and the evolut ion of evolvability. Evolution 50,967-976.

West, G.B., Brown, J.H., 2005. The origin of allometric scaling laws in biology trom genomes to ecosystems: towards a quantitative unifying theory of biological stricture and organization. Journal of Experimental Biology 208 (10t 9). 1575-1592.

West, G.B., Woodruff, W.H., Brown, J., 2002 . Allometric scaling of metabolic rate from molecules and mitochondria to cells and mammals. Proceedings of the National Academy of Science of the USA 99 (Suppl. 1), 2473-2478.

Wilke, C.O., Wang, J.L., Ofria, C., Lenski, R.E., Adami, C., 2001. Evolution of digital organisms at high mutation rates leads to survival of the flattest. Nature 412 (6844), 331-333. 